\title{
Anomalous percolation transition in carbon-black-epoxy composite materials
}

\author{
L. Flandin \\ Centre de Recherche sur les Macromolécules Végétales, CNRS-Université Joseph Fourier, Boîte Postale 53, 38041 Grenoble Cedex, \\ France \\ T. Prasse \\ Polymer/Composites, Technical University Hamburg-Harburg, Denickestrasse, 15, D-21071, Hamburg, Germany \\ and Materials for Microelectronics, Technical University Hamburg-Harburg, Denickestrasse, 15, D-21071, Hamburg, Germany \\ R. Schueler* \\ Polymer/Composites, Technical University Hamburg-Harburg, Denickestrasse, 15, D-21071, Hamburg, Germany \\ and Mechanical and Aerospace Engineering, University of Texas at Arlington, Box 19018, Arlington, Texas 76019 \\ K. Schulte \\ Polymer/Composites, Technical University Hamburg-Harburg, Denickestrasse, 15, D-21071, Hamburg, Germany \\ W. Bauhofer \\ Materials for Microelectronics, Technical University Hamburg-Harburg, Denickestrasse, 15, D-21071, Hamburg, Germany \\ J.-Y. Cavaille ${ }^{\dagger}$ \\ Groupe de Métallurgie Physique et Physique des Matériaux, UMR CNRS 5510, INSA, 69621 Villeurbanne Cedex, France
}

(Received 27 May 1998)

\begin{abstract}
ac and electrical properties of carbon-black-epoxy composites measured in the frequency range of $100 \mathrm{~Hz}$ to $1 \mathrm{MHz}$ are reported. Experimental results are in remarkable disagreement with the predictions of the statistical percolation theory for real and imaginary parts of the conductivity. They both exhibited, in particular, an abrupt variation at a given fraction of carbon black. The evolution of these transport quantities with the volume fraction of carbon black can only be explained considering a phase transition from a dispersed to an agglomerated state of the conducting fillers. This transition has been successfully verified by optical microscopy of thin polished sections of cured samples. A simple model based on electrostatic repulsion between the carbon-black particles is proposed to interpret such a singular insulator-to-conductor transition. [S0163-1829(99)01718-X]
\end{abstract}

\section{INTRODUCTION}

Polymers contain a very low concentration of free charge carriers, and are therefore generally good electrical insulators. However, conducting plastics or thermosets are useful for many applications. ${ }^{1}$ Electrical conductivity can be achieved by incorporation of highly conductive fillers, such as carbon-black (CB) particles, carbon fibers, metallic fillers, or intrinsically conducting polymers.

Electrical properties of such composite materials have been widely studied with both applied and fundamental interest. $^{2}$ The dc conductivity is related to the formation of a network of filler particles within the matrix; it increases sharply at a characteristic conducting particle concentration $\left(V_{c}\right)$ known as the percolation threshold. The percolation transition leads to strong nonlinear properties, but since Kasteleyn and Fortuin ${ }^{3}$ demonstrated that it can be identified with a second-order phase transition, one knows that physical quantities like de conductivity should exhibit a continuous behavior at this transition.

To describe the insulator-to-conductor transition a large variety of models have been proposed in the literature. ${ }^{4,5}$ For several decades the statistical percolation model explained, at least qualitatively, all experimental data. ${ }^{6,18}$ However, as outlined by Carmona, ${ }^{6}$ a full understanding of the electrical and structural properties must involve the specific preparation conditions of the materials, namely, the occurrence of a colloidal phase. This author noticed, in the same review, ${ }^{6}$ that a better description for the structure formation would be useful for a complete and comprehensive understanding of these kinds of materials. The question of "the rules of the game" for the cluster formation has arisen, as well as the influence of the preparation method on the electrical properties of the composite materials. ${ }^{5,7}$ The percolation model proposed by Sumita and co-workers ${ }^{8,9}$ was the first attempt to answer this question by accounting for the filler-matrix interactions. In this thermodynamic model it is assumed that the free interfacial energy of the system $(g)$ cannot exceed a "universal value", $g *$. Thus, the network formation results from the aggregation of the CB particles to prevent the free energy from rising above the $g^{*}$ value. This $g^{*}$ concept allows us to interpret correctly the measurement of different values for the percolation threshold varying the free interfacial energy between filler and matrix.

In a recent review on the models to explain the electrical conductivity of binary mixtures, Lux $^{5}$ pointed out the experi- 
ment performed by Harbourg, Walzak, and Veregin ${ }^{10}$ showing that the mixing process may induce an electrical charging of the $\mathrm{CB}$ particles. Lux noticed that such permanent surface charges might stimulate interactions between $\mathrm{CB}$ particles and the polymeric host. After this review was published, Schueler et al. ${ }^{11}$ assumed that the presence of such an electrical charge at the surface of their black induces particleparticle interactions that could be modified by addition of salt during the thermal curing. Very recently, we have shown, ${ }^{12}$ by applying an electric field on equivalent samples during the curing process, that the carbon particles in these composites do have a permanent negative charge at the surface.

The work described in the present paper deals with the measurement of ac and dc properties of these CB-epoxy composite materials. The goal was to investigate the influence of electrical charges at the surface of conducting fillers on the percolation transition. It is shown that a jump occurs in the conductivity for a given volume fraction of CB. This evolution cannot be described with the statistical percolation model, but has been related to an abrupt change in the microstructure of the composites. This transition of the CB particles from a dispersed to an agglomerated state was measured by ac and dc electrical methods and verified by transmission optical micrographs on cured samples. An interpretation of this behavior is given, based on concepts of colloid science.

\section{THEORY}

\section{A. Percolation}

The most prominent model is the statistical percolation model, proposed by Kirkpatrick ${ }^{13}$ and Zallen. ${ }^{14}$ It allows forecasting both geometric and electrical quantities. It is based on the assumption ${ }^{15}$ that the fillers are not interacting with each other or, in other words, that they are randomly dispersed within the matrix. Under such a condition a binary mixture can be modeled ${ }^{13}$ with a lattice possessing a given fraction of active sites (or bounds) randomly chosen, i.e., independently of the occupation status of their neighbors. Sufficiently close to the transition, the statistical percolation predicts a power-law variation for the measurable quantities with the volume fraction of fillers.

The aim of the statistical percolation theory is to forecast the behavior of a not completely connected set of objects. By varying the number of connections, this model allows us to describe the transition from a local to an infinite "communication" state. The percolation threshold $V_{c}$ is defined as the critical volume fraction separating these two states. The classical theory has proven to be relevant for describing both geometrical and transport properties of composite materials with power-law equations. The correlation length is the average distance between two sites belonging to the same cluster. It is directly related to the cluster formation and reaches very high values on both sides of the transition. Close to the threshold this parameter follows a power-law divergence, ${ }^{15}$ $\xi=\left|V-V_{c}\right|^{-\nu}$, with a value of 0.88 for $\nu$ in threedimensional systems. In the statistical percolation theory the divergence of the correlation length or, in other words, the formation of an infinite cluster, is not due to any agglomera-

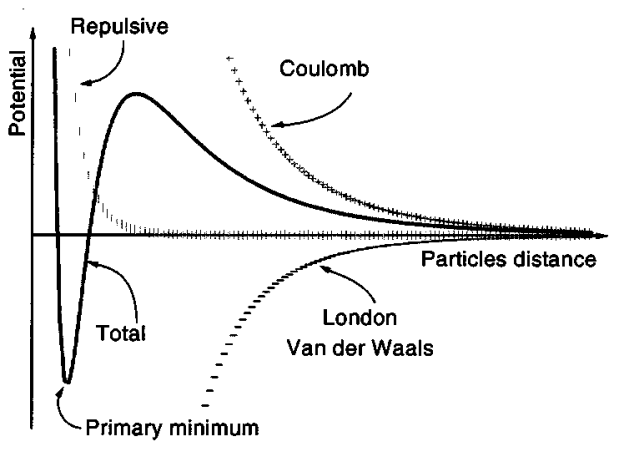

FIG. 1. Potential energy of interaction between two charged particles.

tion, which should involve a driving force and a diffusion process, but to the random position of occupied sites.

The dc electrical conductivity $(\sigma)$ or the dielectric constant $(\varepsilon)$ also exhibit a power-law dependence close to the percolation threshold, ${ }^{4,15}$

$$
\varepsilon \propto\left|V-V_{c}\right|^{-S},
$$

and, for $V>V_{c}$,

$$
\sigma \propto\left(V-V_{c}\right)^{t} .
$$

The exponents $s$ and $t$, characterizing the transition, are assumed to be universal, i.e., to depend only upon the dimensionality of the system. In three-dimensional systems the universal values are $t \cong 2$ and $s \cong 0.7$ for the conductivity ${ }^{16}$ and for the dielectric constant, ${ }^{17,24}$ respectively. Experimentally both universal and nonuniversal values have been proposed ${ }^{18}$ for the exponent $t$. Experimental data reported ${ }^{17,19,20}$ for the exponent $s$ are generally in agreement with Eq. (1).

\section{B. Particle-particle interactions}

Between two fillers in a medium, an attractive Londonvan der Waals potential has to be considered. This interaction is described by a $-1 / r^{6}$ potential, where $r$ is the distance between the particles. Further investigations ${ }^{12}$ on the presently described composites gave direct evidence that the particles' surfaces are negatively charged. Thus, as already postulated by Schueler et al. ${ }^{11}$ and measured by Harbourg, Walzak, and Veregin, ${ }^{10}$ the CB particles possess a permanent electrical charge that leads to a long-range Coulombic repulsion among each other. This Coulombic interaction, described by a $1 / r$ potential, is comparable ${ }^{21}$ to the one commonly used in colloid science ${ }^{22,23}$ to obtain an electrostatic stabilization of dispersions. The total potential between two fillers (Fig. 1) is thus the sum of two competitive potentials plus a repulsion term proportional to $1 / r,{ }^{12}$ which accounts for the contact repulsion.

Due to thermal agitation the particles are moving in the medium. If the collisions created by this Brownian motion involve sufficient energy to overcome the electrostatic barrier, the pair of particles falls in a deep primary minimum of the total potential energy and becomes irreversibly aggregated. In the case of CB-epoxy composites this can also be obtained by shearing the composite before curing. ${ }^{11}$ The height of the barrier depends on the electric charge at the 
surface of the particles. It is also influenced by the ionic strength of the matrices, ${ }^{23}$ which can be altered by the addition of salt. As already presented, ${ }^{11}$ both the shearing and addition of salt leads to a lowering of the conduction threshold in the presently examined composite systems.

\section{EXPERIMENT}

The matrix used in this study was an epoxy polymer obtained from the reaction of a bisphenol- $A$ resin (ARALDIT LY556, CIBA GEIGY) with an aromatic amine hardener (ARALDIT HY932). The filler component was an extra conductive carbon black (PRINTEX XE 2, DEGUSSA AG) that consisted of aggregates composed of spherical particles with a mean diameter of $30 \mathrm{~nm}$. The details of the sample preparation have been described elsewhere. ${ }^{11}$ The first step consists of mixing $500 \mathrm{~g}$ of the resin with the desired amount of $\mathrm{CB}$ for $1 \mathrm{~h}$ at $5000 \mathrm{rpm}$ using a dissolver disk at room temperature. This step induced a good dispersion of the CB particles within the epoxy resin and reduced the size of the $\mathrm{CB}$ agglomerates. After this strong stirring the $\mathrm{CB}$ agglomerates have distributed sizes with a mean diameter in the order of $0.5 \mu \mathrm{m}$. Samples were cured after another stirring of $1 \mathrm{~h}$ at $1000 \mathrm{rpm}$ with the hardener at room temperature. Composites prepared this way will be referred to as " $A$."

To vary the ionic strength of the matrix, another preparation was performed in which $\mathrm{CuCl}_{2}$ (FLUKA) was added and mixed with the hardener. The salt concentration in the resin was adjusted to $3 \times 10^{-6} \mathrm{~mol} / \mathrm{g}$. These samples, prepared in the same conditions as samples $A$, but with an addition of $\mathrm{CuCl}_{2}$, will be referred to as " $B$."

The curing process consisted of heating the mixtures under vacuum conditions for $3 \mathrm{~h}$ at $80^{\circ} \mathrm{C}$ to reach the gel point and for $8 \mathrm{~h}$ at $140^{\circ} \mathrm{C}$ to complete the polymerization of the matrix. Parallelepipedic samples were cut having dimensions of approximately $1 \times 1 \mathrm{~cm}^{2}$ wide and $0.3 \mathrm{~cm}$ thick. Electrical contacts were made of silver paint on opposite sides. The ac electrical measurements were carried out at room temperature with a HP 4284A impedance analyzer with a four-point probe technique. A voltage of $5 \mathrm{~V}$ in amplitude was applied and the complex conductivity $\widetilde{\sigma}$ versus the frequency $f$ was measured in the range of $100 \mathrm{~Hz}$ to $1 \mathrm{MHz}$. The complex dielectric constant $\widetilde{\varepsilon}$ is related to the conductivity by $\widetilde{\varepsilon}$ $=\widetilde{\sigma} / i \omega \varepsilon_{0}$, where $\omega$ denotes the angular frequency and $\varepsilon_{0}$ the dielectric constant of the vacuum. The dc conductivity was measured with a Keithley 617 electrometer. A minimum of three samples has been used to determine the electrical properties for each composition.

Transmission optical micrographs were obtained from thin polished sections of cured composites by using a LEICA POLYVAR microscope.

\section{RESULTS AND DISCUSSION}

This section describes first the electrical conductivity measured versus the frequency for different amounts of CB. Second, it will be shown that the evolution of real and imaginary parts of the conductivity versus filler content is in clear disagreement with the prediction of the statistical percolation theory. Third, an attempt to explain such a departure is proposed, which is based on the evolution of the filler arrange-

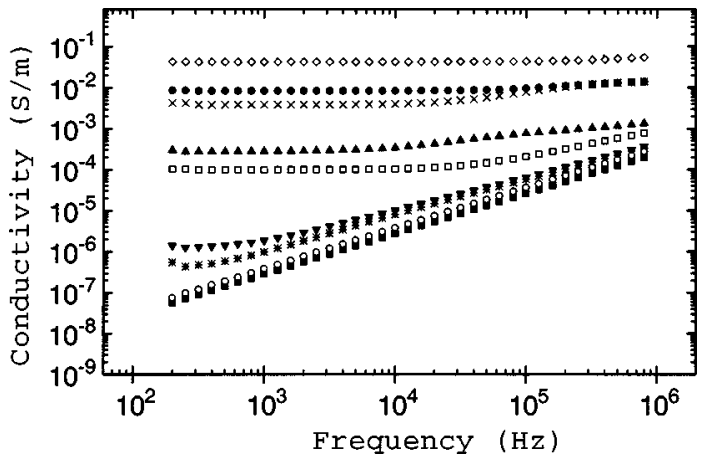

FIG. 2. Frequency dependence of the conductivity for $0(\mathbf{\square})$, $0.012(\bigcirc), 0.03(*), 0.06(\boldsymbol{\nabla}), 0.12(\square), 0.3(\mathbf{\Delta}) ; 0.48(\times), 0.6(\bullet)$, and $0.9(\diamond)$ volume percent of carbon black with addition of $\mathrm{CuCl}_{2}$.

ment within the matrix. This assumption is, finally, verified with transmission optical micrographs of the cured sample.

\section{A. Frequency dependence}

Figure 2 displays the frequency dependence of the electrical conductivity for the salt-agglomerated samples $B$. These composites exhibited a typical dielectric behavior below the conduction threshold, i.e., the conductivity increased linearly with the frequency while the phase angle was close to $\pi / 2$. Above this threshold of $0.03 \%$ a finite conductivity led to a plateau at low frequency corresponding to the electrical response of the percolating network. The conductivity increased at higher frequencies due to the growing importance of the polarization effects on the macroscopic conductivity. Increasing the volume fraction of fillers increased the low-frequency conductivity and led to a broader plateau. Similar results have frequently been reported ${ }^{19,20,24}$ and related to the formation of a percolating network in the material. With composites $B$ each point of the conductivityfrequency plane seems to be reachable, by choosing the right volume fraction of fillers. The insulator-to-conductor transition appears continuous.

On the contrary, in the case of samples $A$ (Fig. 3) a jump in the conductivity at the conduction threshold of $0.9 \%$ was measured. While a purely dielectric behavior was also observed below this threshold, the conductivity was almost independent of the frequency above this critical volume frac-

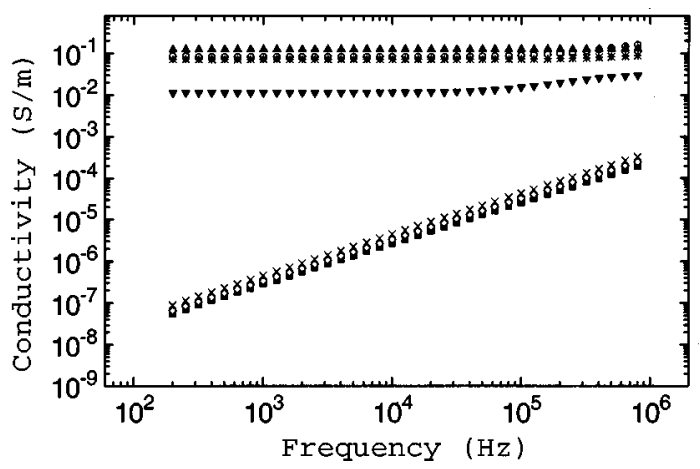

FIG. 3. Frequency dependence of the conductivity for $0(\mathbf{\square}), 0.6$ $(\diamond), 0.9(\times), 0.93(\boldsymbol{\nabla}), 0.96(*), 1.05(\bigcirc)$, and $1.2(\mathbf{\Delta})$ volume percent of $\mathrm{CB}$ without addition of $\mathrm{CuCl}_{2}$. 


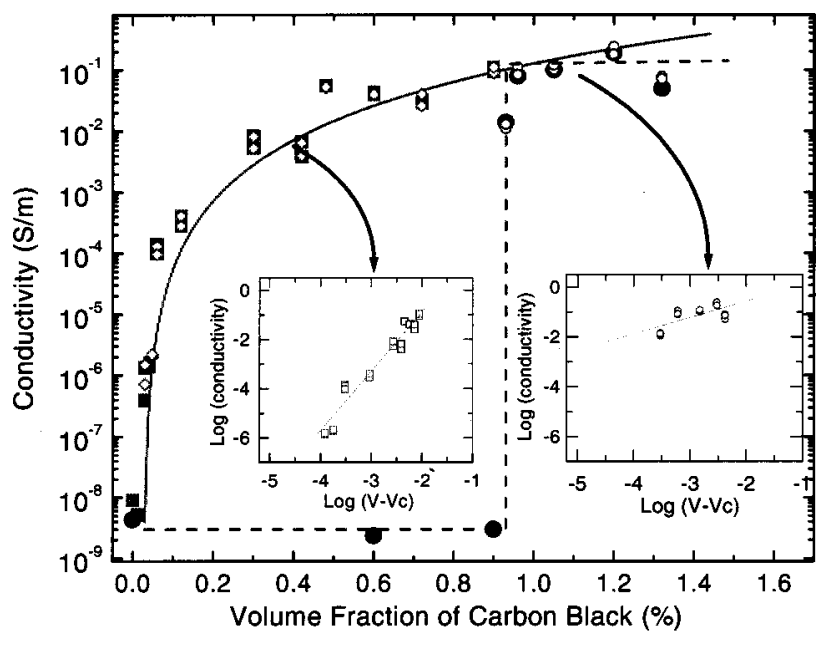

FIG. 4. Real part of the conductivity at $1 \mathrm{kHz}$ with $(\boldsymbol{\square})$ and without $(\bullet)$ addition of $\mathrm{CuCl}_{2}$. dc electrical properties with $(\diamond)$ and without $(\bigcirc)$ addition of $\mathrm{CuCl}_{2}$. Insets: comparison with powerlaw dependencies.

tion where conduction took place. Moreover, there was a large gap between the conductivity measured on both sides of the transition. Nevertheless, for similar amounts of CB above $0.9 \%$, the frequency dependencies of the conductivity for the samples $A$ and $B$ are comparable.

\section{B. Real and imaginary part of the conductivity}

Figure 4 displays the real part of the conductivity measured at $1 \mathrm{kHz}$ as well as the dc conductivity for both sets of samples. ${ }^{25}$ The values of the dc conductivity were found very close to that of the ac conductivity at $1 \mathrm{kHz}$ as already described in the literature. ${ }^{26}$ As already mentioned, material $B$ exhibited a continuous increase in the conductivity with the $\mathrm{CB}$ content. The variation of the conductivity with the volume fraction of filler was in good agreement with the powerlaw equation (2) and the critical exponent $t$ was found to be $2.4 \pm 0.2$ (inset of Fig. 4). Such a value is compatible with the predictions of the statistical percolation theory, even if nonuniversal.

Samples $A$ remained good insulators up to a volume fraction of $0.9 \%$. This defined accurately the conduction threshold as an increase of the CB content as low as $0.03 \%$ led to a jump in the conductivity of about seven orders of magnitude. The conductivity reached values that were surprisingly close to those obtained for type- $B$ composites. Increasing the volume fraction of $\mathrm{CB}$ above $0.93 \%$ induced a minor rise in the conductivity. Such a behavior could not be fitted properly with Eq. (2) and gave anyway a value of 0.64 for $t$ (inset of Fig. 4), which is in strong disagreement with the statistical percolation theory.

The relative permittivity at low frequency $(1 \mathrm{kHz})$ for these two sets of composites is depicted in Fig. 5. From this graph, it is evident that this quantity is, again, in disagreement with the percolation theory, which predicts [Eq. (1)] a divergence in the permittivity close to the percolation threshold. Indeed, the dielectric constant in the case of composites $B$ increased above the conduction threshold and has a variation comparable with the one of the real part of the conductivity.

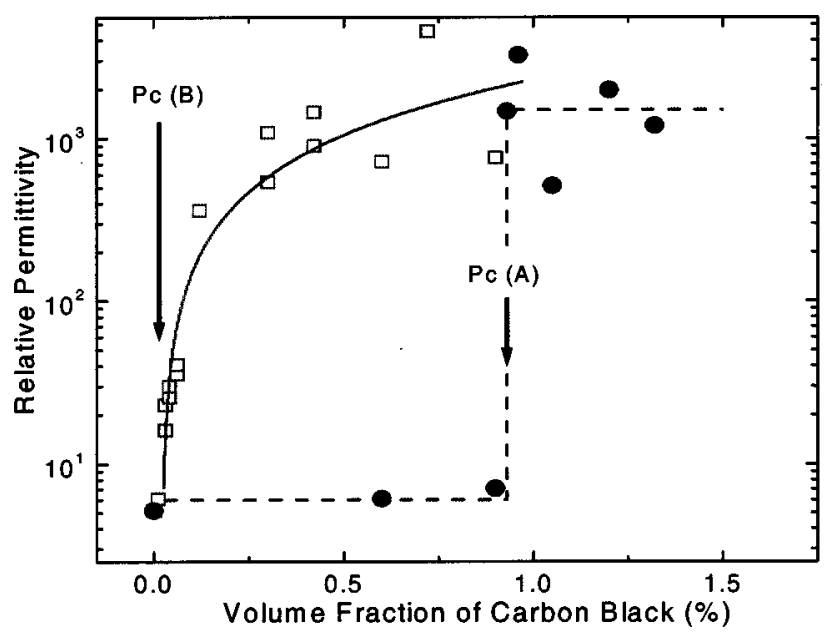

FIG. 5. Dielectric constant at $1 \mathrm{kHz}$ versus $\mathrm{CB}$ content for composites processed with $(\square)$ and without $(\bullet)$ addition of $\mathrm{CuCl}_{2}$.

For samples $A$ almost no change in the dielectric constant was measured by varying the volume fraction of $\mathrm{CB}$ below the conduction threshold. This observation is very important for the understanding of the microstructure in the materials. Following scaling theory, ${ }^{15}$ the divergence of the permittivity is related to the divergence of the correlation length. A sharp increase in the latter with the volume fraction of filler is a quite normal behavior for a random composite material approaching the transition. The constant value for the permittivity was, in the present case, interpreted as due to the Coulombic filler-filler repulsion that disturbs the cluster formation. At the threshold another discontinuity was measured for the permittivity: a change of $0.03 \%$ of the $\mathrm{CB}$ content induced an evolution of two orders in magnitude for $\varepsilon$ that seemed to arise from the network formation.

\section{Interpretation of the transitions}

The increase in the relative permittivity slightly above the conduction thresholds for both sets of samples was contrary to the decrease predicted by the percolation theory [Eq. (1)] for randomly dispersed composites. Moreover, samples $B$ exhibited a continuous increase for the real part of the conductivity, but it was not possible to process samples of type $A$ with an intermediate conductivity, because it was not possible to weigh $\mathrm{CB}$ and epoxy resin so as to obtain accurately $0.915 \%$ of $\mathrm{CB}$ content. It was evident anyway that this behavior could not be explained by the classical theory for binary insulator-conductor mixtures, but that the dynamics of the CB fillers in the polymer host had to be considered.

At the end of the processing (i.e., after strong stirring), no conducting network exists in the composite, whatever the salt concentration. This has been shown in a previous study ${ }^{11}$ by measuring the conductivity versus the time of the curing process: the initial conductivity always exhibited a very low value.

An increase in the ionic concentration of the epoxy resin lowers the potential barrier among the $\mathrm{CB}$ particles in samples $B$. Thus, the fillers will form easily clusters from this initial state, with the support of the Brownian motion. A three-dimensional network will be created and, accordingly, a finite conductivity. Because of this kind of cluster forma- 
0.9 \% Carbon Black, with $\mathrm{CuCl}_{2}$ added

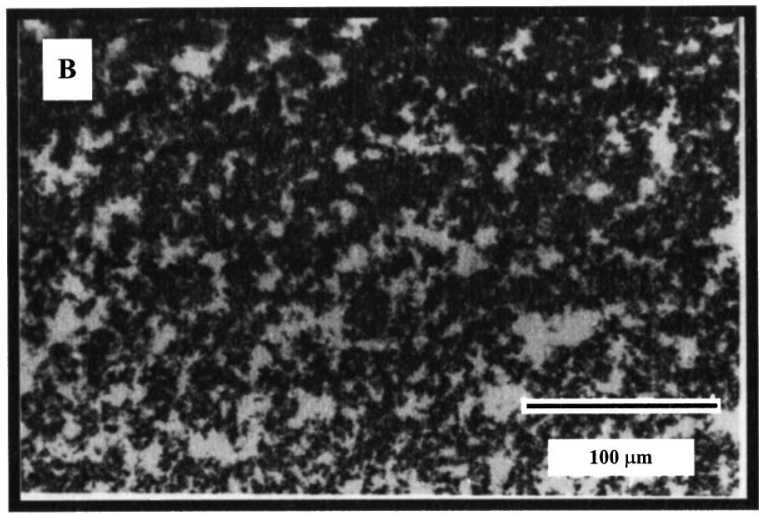

$0.9 \%$ Carbon Black, without $\mathrm{CuCl}_{2}$ added

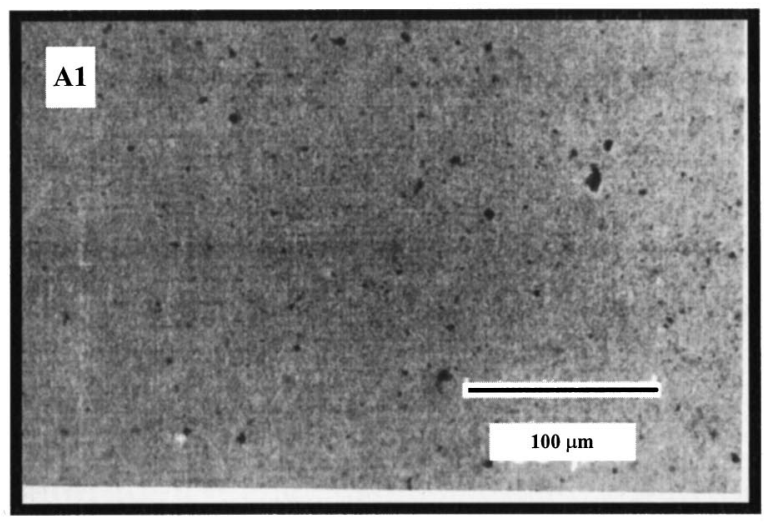

$0.93 \%$ Carbon Black, without $\mathrm{CuCl}_{2}$ added

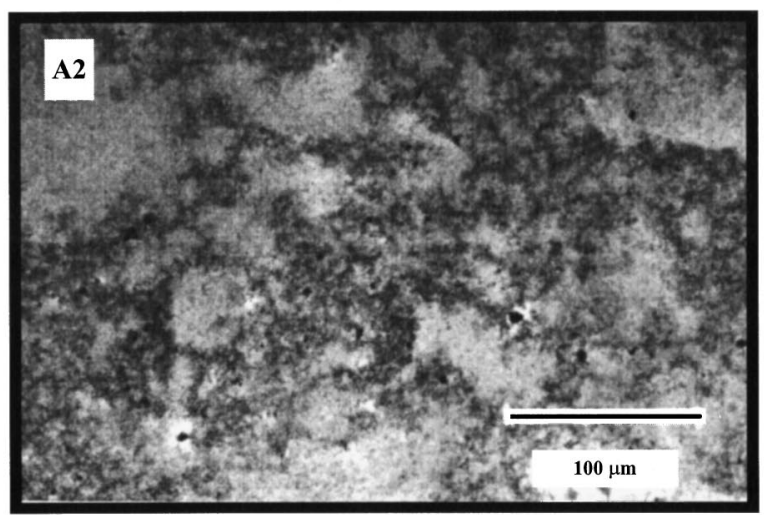

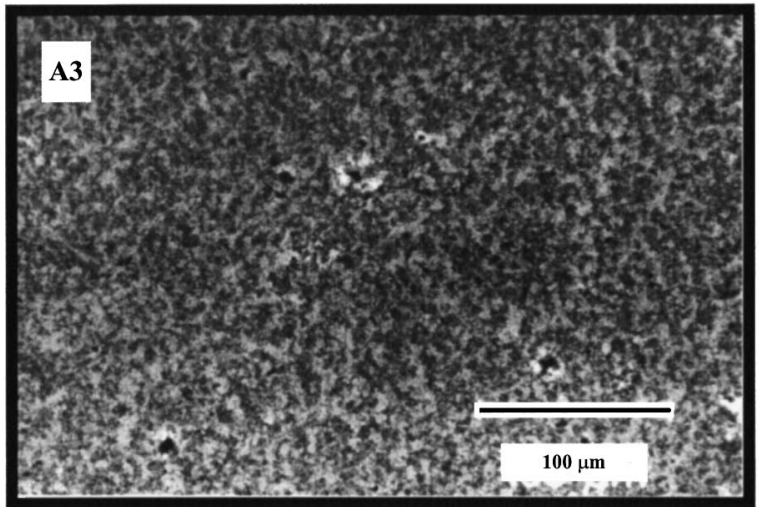

FIG. 6. Transmission optical micrographs of thin polished sections of cured material, (B) $0.9 \%$ with $\mathrm{CuCl}_{2}$ and (A1) $0.9 \%$, (A2) $0.93 \%$, and (A3) $0.96 \%$ without $\mathrm{CuCl}_{2}$.

tion, a strongly agglomerated structure will be achieved and thereby a very low conduction threshold, compared with the standard percolation theory and most of the results proposed in the literature. The final microstructures of these composites are thus not random [Fig. 6(B)] and this is most likely the reason why the permittivity increases with the $\mathrm{CB}$ content.

More interesting are the $A$ samples, prepared without the addition of salts. For a volume fraction of $\mathrm{CB}$ less than or equal to $0.9 \%$, the particles repel each other and try to move as far away as possible to decrease their potential energy of interaction. This electrostatic stabilization prevents the formation of clusters and thereby leads to a weak correlation length and to a low permittivity. Nevertheless, by increasing the volume fraction of $\mathrm{CB}$, the particles must reach a closer position. Exceeding this critical value of $\mathrm{CB}$ content leads to composites with "high" conductivity. This high value is, in fact, comparable to the one obtained with a strongly agglomerated composite (type $B$ ) with an equivalent $\mathrm{CB}$ content indicating an analogous degree of formation for the percolating network.

In summary this material changes singularly, with a variation of CB content of only $0.03 \%$, from a good insulating behavior with a low permittivity (comparable to that of the pure matrix) to a rather good conducting behavior equivalent to that of a totally agglomerated composite. It is evident that such an evolution should be related to an important modification in the filler arrangement within the polymer host. If true, this assumption should induce a strong variation in the microstructure of the composites. In a previous paper ${ }^{12}$ we have shown that the transmission optical microscopy was relevant to describe the cluster formation of these composites. In situ measurements of the conductivity of a sample placed in an optical microscope during the curing process permitted us to correlate directly the dispersion state with the level of conductivity. We concluded that the samples initially insulating and with an even dispersion of the filler become conducting during the curing with appearance of strong heterogeneity in the microstructure.

In the present case, to check the existence of an abrupt change in the microstructure of the material, optical micrographs of thin polished sections of the cured materials were taken (Fig. 6). The first micrograph (B) shows a sample with addition of salt, containing $0.9 \%$ of $\mathrm{CB}$. In this case all the carbon particles are agglomerated leading to large particlefree areas. This is in agreement with the previous study ${ }^{11}$ that showed that a strong agglomeration always occurred in the $B$ samples, even for much lower CB contents. In fact, the microstructure of the $B$ materials cannot be compared to the one 
obtained with a random occupation of sites of a given lattice, and therefore its behavior cannot be compared directly with a statistical percolation.

The second picture (A1) corresponds to the same volume fraction of $0.9 \%$ of $\mathrm{CB}$, it reveals evenly distributed $\mathrm{CB}$ particles and, as expected, no visible cluster formation took place in the composite. This microstructure was the same at different places of the sample. On the contrary, for a volume fraction of $\mathrm{CB}$ of $0.93 \%$ (A2), i.e., just above the measured conduction threshold, an aggregation phenomenon can be observed leading to an inhomogeneous distribution of the filler in the material. Indeed, particle-free areas and enriched zones, which favor the electrical conduction, can be seen on this picture. However, the hardening of the epoxy resin stopped the process before all particles where agglomerated and both aggregated and dispersed areas can be found in this sample by varying the observed area. The last picture (A3), obtained for a volume fraction equal to $0.96 \%$ of $\mathrm{CB}$, shows a highly structured network, similar to the one obtained for samples type $B$ and thereby explaining the equivalent conductivity measured on these two kinds of samples. This agglomerated structure is found in the whole sample.

These observations are in very good agreement with the electrical measurements and confirmed ${ }^{12}$ that insulating composites are to related to a dispersed state of the fillers while conducting composites should be associated with an agglomerated state of the fillers. These pictures contributed, thereby, to explain the abrupt conduction transition measured in the type- $A$ composites.

\section{CONCLUSIONS}

This work has dealt with ac and dc electrical properties of strongly contrasted binary mixtures made of an epoxy resin and CB particles. The experimental results showed a marked difference with the predictions of the statistical percolation theory available for randomly dispersed fillers. The measurements could, however, clearly be related to a nonrandom microstructure observed by transmission optical microscopy. The dynamics of the filler in the epoxy resin during the curing process, responsible for these microstructures, could be qualitatively explained with the help of the concepts of colloid science.

The low dielectric constant in the vicinity (but below) the conduction threshold in the case of the composites $A$ indicates clearly that no cluster formation takes place just before the transition. This can be understood by the electrostatic stabilization of the particles. An abrupt phase transition at a critical volume fraction of $0.9 \%$ could be explained as a consequence of a transition in the filler arrangement within the matrix. The critical value of $\mathrm{CB}$ content separates, in fact, a dispersed and an agglomerated state and can therefore not be compared with the one obtained through a purely random geometrical process.

This work confirms the necessity of accounting for the structure formation for the understanding of the percolative behavior of such binary mixtures. In the present study the $\mathrm{CB}$ particles have permanent electrical charges at their surfaces. It is, however, not certain that this phenomenon, particularly striking with the present composites, does not exist in other kinds of heterogeneous materials.

\section{ACKNOWLEDGMENTS}

We wish to thank Dr. H. Wittich and Dr. M. Kupke for the stimulating discussions during this work. DAADPROCOPE is also gratefully acknowledged for the financial support of the research collaboration program. One of us (L.F.) also thanks the Région Rhône Alpes for financial support.
*Deceased.

†Present address. Author to whom correspondence should be addressed.

${ }^{1}$ A. T. Ponomarenko, V. G. Schevchenko, and N. S. Enykolopyan, Adv. Polym. Sci. 96, 125 (1990).

${ }^{2}$ J. S. Andrade, N. Ito, and Y. Shibusa, Phys. Rev. B 54, 3910 (1996).

${ }^{3}$ P. W. Kasteleyn and C. M. Fortuin, J. Phys. Soc. Jpn. 26, 11 (1969).

${ }^{4}$ J. P. Clerc, G. Giraud, J. M. Laugier, and J. M. Luck, Adv. Phys. 39, 191 (1990).

${ }^{5}$ F. Lux, J. Mater. Sci. 28, 285 (1993).

${ }^{6}$ F. Carmona, Physica A 157, 461 (1989).

${ }^{7}$ L. Karásek and M. Sumita, J. Mater. Sci. 31, 281 (1996).

${ }^{8}$ K. Miyasaka, K. Watanabe, E. Jojima, H. Aida, M. Sumita, and K. Ishikawa, J. Mater. Sci. 17, 1610 (1982).

${ }^{9}$ M. Sumita, H. Abe, H. Kayaki, and K. Miyasaka, J. Macromol. Sci., Phys. B25, 171 (1986).

${ }^{10}$ J. R. Harbourg, M. J. Walzak, and R. P. Veregin, J. Colloid Interface Sci. 138, 380 (1990).

${ }^{11}$ R. Schueler, J. Petermann, K. Schulte, and H. P. Wentzel, J. Appl. Polym. Sci. 63, 1741 (1997); R. Schueler, Ph.D. thesis, Technical University Hamburg-Harburg, 1994.

${ }^{12}$ T. Prasse, L. Flandin, K. Schulte, and W. Bauhofer, Appl. Phys. Lett. 72, 2903 (1998)
${ }^{13}$ S. Kirkpatrick, Rev. Mod. Phys. 45, 574 (1973).

${ }^{14}$ R. Zallen, The Physics of Amorphous Solids (Wiley, New York, 1983), Chap. 4.

${ }^{15}$ D. Stauffer and A. Aharony, Introduction to Percolation Theory (Taylor \& Francis, Washington, D.C., 1992).

${ }^{16}$ B. Derrida, D. Stauffer, H. J. Hermann, and J. Vannemius, J. Phys. (France) Lett. 44, L701 (1983).

${ }^{17}$ Y. Song, T. W. Noh, S. I. Lee, X. D. Chen, and J. R. Gaines, Phys. Rev. B 33, 904 (1986); A. B. Harris, Phys. Rev. Lett. 54, 2614 (1989).

${ }^{18}$ M. B. Heaney, Phys. Rev. B 52, 12477 (1995).

${ }^{19}$ J. Yacubowicz and M. Narkis, Polym. Eng. Sci. 26, 1568 (1986).

${ }^{20}$ T. A. Ezquerra, M. Mohammadi, F. Kremer, T. Vilgis, and G. Wegner, J. Phys. C 21, 927 (1988).

${ }^{21}$ This $1 / r$ potential, available for ion-ion interaction, is the good one for the present study where the particles possess a permanent electrical charge. A $1 / r^{2}$ potential, is used in colloid science to describe dipole-dipole interaction (see Ref. 22).

${ }^{22}$ See, for example, D. F. Evans and H. Wennerström, The Colloidal Domain: Where Physics, Chemistry, Biology and Technology Meet (VCH, New York, 1994); or D. J. Shaw, Introduction to Colloid Surface Chemistry, 3rd Ed. (Butterworth-Heinemann, London, 1980).

${ }^{23}$ E. J. Verwey and J. T. G. Overbeek, Theory of the Stability of 
Lyophobic Colloids (Elsevier, Amsterdam, 1948).

${ }^{24}$ T. A. Ezquerra, M. Kulescza, and F. J. Baltà-Calleja, Synth. Met. 41, 915 (1991).

${ }^{25}$ The real part of conductivity could not be measured accurately below the conduction thresholds, as the phase angle was close to $\pi / 2$. A value in the order of $10^{-9} \mathrm{~S} / \mathrm{m}$ overestimates the real conductivity of the matrix, which was found with another setup (Ref. 11) to be close to $10^{-12} \mathrm{~S} / \mathrm{m}$.

${ }^{26}$ M. T. Connor, S. Roy, T. A. Ezquerra, and F. J. Baltá Calleja, Phys. Rev. B 57, 2286 (1998). 